\title{
Charge-Exchange Reactions with a Radioactive Triton Beam
}

\author{
J. Jänecke ${ }^{\dagger}$ \\ University of Michigan, Ann Arbor, Michigan 48109-1120, U.S.A.
}

\begin{abstract}
A high-resolution $\left(\mathrm{t},{ }^{3} \mathrm{He}\right)$ test experiment has been performed recently by making use of a secondary triton beam produced by fragmentation of $\alpha$-particles. The purpose of this charge-exchange experiment was to achieve good energy resolution in an $(\mathbf{n}, \mathbf{p})$-type reaction at intermediate bombarding energies. The experiment was carried out with the K1200 cyclotron at the National Superconducting Cyclotron Laboratory using the A1200 beam-analysis system and the S800 magnetic spectrometer. The beam-analysis system was used to transport the energy-dispersed radioactive triton beam from the production target to the target position, and the magnetic spectrometer was used to focus the dispersion-matched ${ }^{3} \mathrm{He}$ particles from the $\left(\mathrm{t},{ }^{3} \mathrm{He}\right)$ reaction at $0^{\circ}$ onto the focal plane of the spectrometer. An energy resolution of $200-250 \mathrm{keV}$ was achieved.
\end{abstract}

Charge-exchange reactions selectively excite isovector non-spinflip and spinflip states and giant resonances. The $(p, n)$ and other $(p, n)$-like reactions, e.g. $\left({ }^{3} \mathrm{He}, t\right)$ or $\left({ }^{6} \mathrm{Li},{ }^{6} \mathrm{He}\right)$, have been studied for many years and much information on spin-isospin excitations of nuclear states has become available for proton-rich nuclei. More recently, particularly the $\left({ }^{3} \mathrm{He}, \mathrm{t}\right)$ reaction with its good energy resolution has added important data. Less information exists for neutron-rich nuclei from the (n,p) and $(n, p)$-like reactions. This is due to the fact that it is necessary to use a secondary neutron beam in ( $n, p)$ reactions, and the energy resolution is more limited. Other (n,p)-like reactions, e.g. $\left(\mathrm{d},{ }^{2} \mathrm{He}\right),\left(\mathrm{t},{ }^{3} \mathrm{He}\right),\left({ }^{7} \mathrm{Li},{ }^{7} \mathrm{Be}\right)$, and $\left({ }^{7} \mathrm{Li},{ }^{7} \mathrm{Be} \gamma\right)$, require coincidence measurements or a secondary beam as well. Whereas the $\left(t,{ }^{3} \mathrm{He}\right)$ reaction does require a secondary beam, it has the potential of good energy resolution because of the expected good beam quality compared to neutron beams in conjunction with the use of magnetic spectrometers. A primary triton beam is in principle possible, though, but there is presently no accelerator facility in existence with a dedicated tritium ion source to accelerate ions to intermediate energies of $>100 \mathrm{~A} \cdot \mathrm{MeV}$.

Spin-isospin excitations in nuclei are of fundamental interest and have been studied for a long time both experimentally and theoretically. The nuclear responses for the operators $\sigma \tau$ and $\sigma \tau r Y_{1}$ have attracted special attention. These operators determine the Gamow-Teller and first-forbidden $\beta$-decay matrix elements. Gamow-Teller

CP455, ENAM98: Exotic Nuclei and Atomic Masses

edited by B. M. Sherrill, D. J. Morrissey, and Cary N. Davids

(C) 1998 The American Institute of Physics 1-56396-804-5/98/ $\$ 15.00$ 
quenching is apparently still not fully understood. These matrix elements are also of basic interest in many astrophysical studies including supernovae explosions and in questions related to double $\beta$-decay and the detection of neutrinos.

$(\mathrm{n}, \mathrm{p})$-like charge-exchange reactions permit the investigation of neutron-rich nuclei including light neutron-halo nuclei. Good energy resolution is especially desirable in most applications of basic nuclear physics and astrophysics. It is concluded from the present work that $\left(t,{ }^{3} \mathrm{He}\right)$ charge-exchange reactions with good energy resolution will become competitive with $(n, p)$ reactions as a tool to study spin-isospin excitations in neutron-rich nuclei including investigations of low-lying Gamow-Teller strength and the general characteristics of spin dipole, quadrupole and monopole resonances.

The recent successful attempt to use the $\left(t,{ }^{3} \mathrm{He}\right)$ charge-exchange reaction to achieve high energy resolution (1) in an ( $n, p)$-type reaction at intermediate bombarding energies and at $0^{\circ}$ is a continuation of earlier work $(2,3)$ which was initiated a few years ago. Here, a secondary triton beam was produced by fragmentation of $\alpha$-particles on a thick Be production target. The first stage of the A1200 beamanalysis system was used to direct the energy-dispersed triton beam to the target at an intermediate dispersive image. The second stage was used to focus the dispersionmatched ${ }^{3} \mathrm{He}$ particles from the $(t, \mathrm{He})$ reaction onto the focal plane. An energy resolution of $780 \mathrm{keV}$ was achieved (2,3). Experimental details are given in Ref. (2). The investigation of Gamow-Teller strengths from the $\left(t,{ }^{3} \mathrm{He}\right)$ reaction on targts of ${ }^{9} \mathrm{Be},{ }^{10} \mathrm{~B},{ }^{11} \mathrm{~B},{ }^{12} \mathrm{C}$, and ${ }^{13} \mathrm{C}$ has been described in Ref. (3).

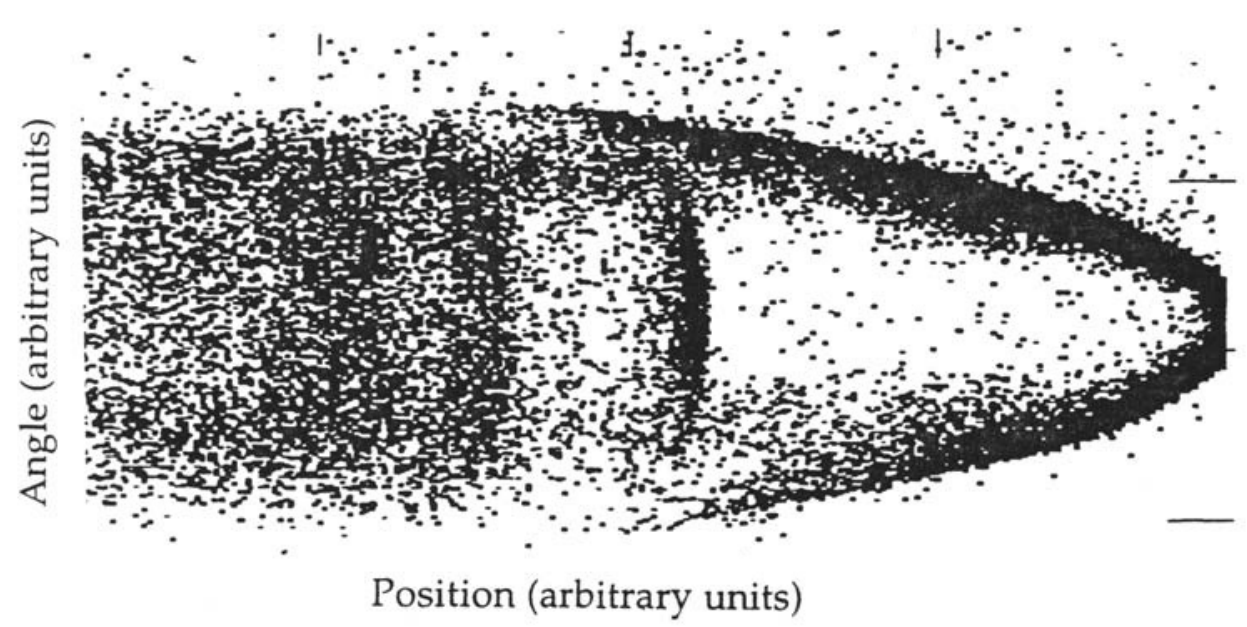

FIGURE 1. Plot of angle versus position for the polyethylene target (online). The events along the strongly curved line are from hydrogen. The essentially vertical lines are from ${ }^{12} \mathrm{C}$. 
The improved $\left(t,{ }^{3} \mathrm{He}\right)$ test experiment (1) was performer by groups of researchers from the National Superconducting Cyclotron Laboratory, the Research Center for Nuclear Physics, the Indiana University Cyclotron Facility, Osaka University, the Kernfysisch Versneller Instituut, and the University of Michigan. It made use for the first time of the $\mathrm{S} 800 \mathrm{high}$-resolution magnetic spectrometer. The triton beam was produced by fragmentation of $560-\mathrm{MeV} \alpha$-particles, slightly lower in energy than in the previous experiments, on a thick Be production target $\left(9.25 \mathrm{~g} / \mathrm{cm}^{2}\right)$. A beam intensity of $(0.5-1.0) \times 10^{6}$ tritons/s was obtained. The energy-dispersed triton beam of median energy $350 \mathrm{MeV}$ or $117 \mathrm{~A} \cdot \mathrm{MeV}$, also slightly lower in energy than previously, was transported to the $\mathrm{S} 800$ target position using the $\mathrm{A} 1200$ beam analyzer. About $1 \%$ of the energy spread of the secondary triton beam was transmitted. This resultsd in a $5 \mathrm{~cm}$ tall beam spot at the target position. Dispersion matching was employed to obtain ${ }^{3} \mathrm{He}$ energy spectra with excellent energy resolution in the focal plane of the $\mathrm{S} 800$ magnetic spectrometer. The focal-plane detection system consisted of two cathode readout drift chambers (CRDC) and two scintillators. An energy resolution of $200-250 \mathrm{keV}$ was achieved for the ground state of ${ }^{12} \mathrm{~B}$ with a polystyrene target of thickness $\rho \Delta \mathrm{x} \approx 5.5 \mathrm{mg} / \mathrm{cm}^{2}$. Reactions on the hydrogen component of the target did not interfere with the reactions on carbon. In fact, the intersection between the loci for $\mathrm{p}\left(\mathrm{t},{ }^{3} \mathrm{He}\right) \mathrm{n}$ and ${ }^{12} \mathrm{C}\left(\mathrm{t},{ }^{3} \mathrm{He}\right){ }^{12} \mathrm{~B}_{g s}$ in the angle versus

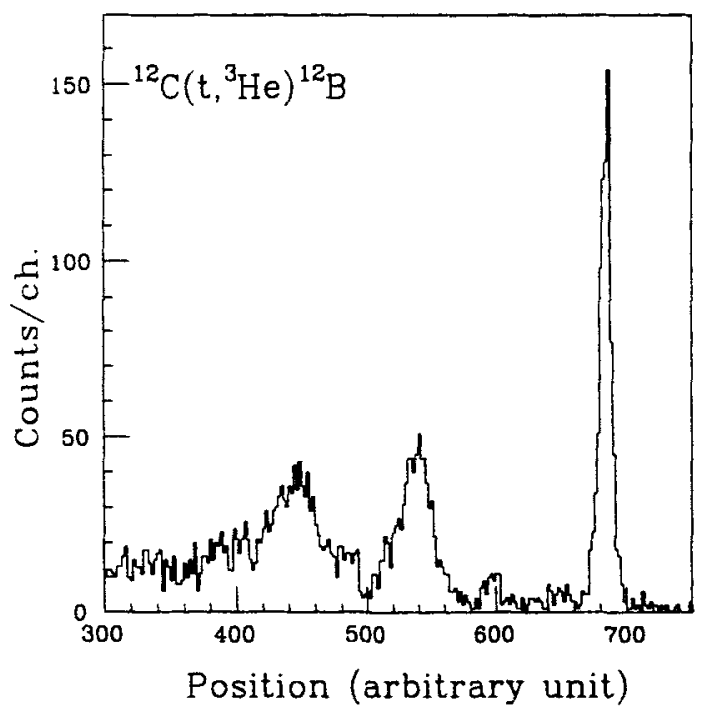

FIGURE 2. Spectrum for angles close to $0^{\circ}$ for the ${ }^{12} \mathrm{C}\left(\mathrm{t},{ }^{3} \mathrm{He}\right){ }^{12} \mathrm{~B}$ reaction at 117 $\mathrm{A} \cdot \mathrm{MeV}$ (preliminary). The energy resolution for the transition to the ground state is 200$250 \mathrm{keV}$. The other two strong peaks are for the resonances at $\sim 4.5 \mathrm{MeV}$ and $\sim 7.5 \mathrm{MeV}$, respectively. 
position plane, as shown in Fig. 1, provides a convenient angle calibration. The weak curvature for the ${ }^{12} \mathrm{~B}$ lines is easily removed in the offline analysis. Figure 2 displays a preliminary spectrum for angles close to $0^{\circ}$. The energy resolution for the ground state is $<250 \mathrm{keV}$. The two other strong peaks are the known $2^{-} / 4^{-}$and $1^{-} / 2^{-}$resonances at $\sim 4.5$ and $\sim 7.5$, respectively. Additional weak states are also seen.

A nickel target was also bombarded in the present work to obtain initial information about low-lying Gamow-Teller strength. The GT + states are found in neutron-rich nuclei at low excitation energies due to the attractive contributions in the particle-particle interaction.

\section{ACKNOWLEDGMENTS}

This work was supported in part by the U.S. National Science Foundation, by the Ministry of Education, Science, Sports, and Culture of Japan (Monbusho), and by the Stichting voor Fundamenteel Onderzoek der Materie (FOM), the Netherlands.

\section{REFERENCES}

† For the collaboration.

1. Sherrill, B. M., Akimune, H., Alahari, N., Austin, Sam M., Bazin, D., van den Berg, A., Berg, G. P. A., Caggiano, J., Daito, I., Fujimura, H., Fujita, Y., Fujiwara, M., Harakeh, M. N., Jänecke, J., Roberts, and D. A., Steiner, M., (to be published).

2. Daito, I., Akimune, H., Austin, S. M., Bazin, D., Berg, G. P. A., Brown, J. A., Davids, B. S., Fujita, Y., Fujimura, H., Fujiwara, M., Hazama, R., Inomata, T., Ishibashi, K., Jänecke, J., Nakayama, S., Pham, K., Roberts, D. A., Sherrill, B. M., Steiner, M., Tamii, A., Tanaka, M., Toyokawa, H., and Yosoi, M., Nucl. Instr. Meth. A 397, 465-471 (1997).

3. Daito I., et al., Phys. Lett. B418, 27-33 (1998). 